\title{
Hydrocephalus treatment in patients with craniosynostosis: an analysis from the Hydrocephalus Clinical Research Network prospective registry
}

\author{
Christopher M. Bonfield, MD, ${ }^{1}$ Chevis N. Shannon, MBA, MPH, DrPH, Ron W. Reeder, PhD, ${ }^{2}$ \\ Samuel Browd, MD, PhD, ${ }^{3}$ James Drake, MBBCh, MSc, ${ }^{4}$ Jason S. Hauptman, MD, PhD, ${ }^{3}$ \\ Abhaya V. Kulkarni, MD, PhD, ${ }^{4}$ David D. Limbrick Jr., MD, PhD, ${ }^{5}$ Patrick J. McDonald, MD, MHSc, ${ }^{6}$ \\ Robert Naftel, MD, Ian F. Pollack, MD, Jay Riva-Cambrin, MD, MSc, ${ }^{8}$ Curtis Rozzelle, MD, ${ }^{9}$ \\ Mandeep S. Tamber, MD, PhD, ${ }^{6}$ William E. Whitehead, MD, MPH, ${ }^{10}$ John R. W. Kestle, MD, ${ }^{11}$ and \\ John C. Wellons III, MD, MSPH, ${ }^{1}$ for the Hydrocephalus Clinical Research Network (HCRN)
}

'Department of Neurosurgery, Vanderbilt University Medical Center, Nashville, Tennessee; Departments of 2Pediatrics and ${ }^{11}$ Neurosurgery, University of Utah, Salt Lake City, Utah; ${ }^{3}$ Department of Neurosurgery, University of Washington, Seattle, Washington; ${ }^{4}$ Division of Neurosurgery, University of Toronto, Ontario, Canada; ${ }^{5}$ Department of Neurosurgery, Washington University School of Medicine in St. Louis, Missouri; ${ }^{6}$ Division of Neurosurgery, University of British Columbia, Vancouver, British Columbia, Canada; ${ }^{7}$ Department of Neurosurgery, University of Pittsburgh Medical Center, Pittsburgh, Pennsylvania; ${ }^{8}$ Division of Neurosurgery, University of Calgary, Alberta, Canada; ${ }^{9}$ Department of Neurosurgery, University of Alabama at Birmingham School of Medicine, Birmingham, Alabama; and ${ }^{10}$ Department of Neurosurgery, Baylor College of Medicine, Houston, Texas

OBJECTIVE Hydrocephalus may be seen in patients with multisuture craniosynostosis and, less commonly, singlesuture craniosynostosis. The optimal treatment for hydrocephalus in this population is unknown. In this study, the authors aimed to evaluate the success rate of ventriculoperitoneal shunt (VPS) treatment and endoscopic third ventriculostomy (ETV) both with and without choroid plexus cauterization (CPC) in patients with craniosynostosis.

METHODS Utilizing the Hydrocephalus Clinical Research Network (HCRN) Core Data Project (Registry), the authors identified all patients who underwent treatment for hydrocephalus associated with craniosynostosis. Descriptive statistics, demographics, and surgical outcomes were evaluated.

RESULTS In total, 42 patients underwent treatment for hydrocephalus associated with craniosynostosis. The median gestational age at birth was 39.0 weeks (IQR 38.0, 40.0); $55 \%$ were female and $60 \%$ were White. The median age at first craniosynostosis surgery was 0.6 years (IQR $0.3,1.7)$, and at the first permanent hydrocephalus surgery it was 1.2 years (IQR 0.5, 2.5). Thirty-three patients (79\%) had multiple different sutures fused, and 9 had a single suture: 3 unicoronal $(7 \%), 3$ sagittal $(7 \%), 2$ lambdoidal (5\%), and 1 unknown (2\%). Syndromes were identified in 38 patients $(90 \%)$, with Crouzon syndrome being the most common $(n=16,42 \%)$. Ten patients $(28 \%)$ received permanent hydrocephalus surgery before the first craniosynostosis surgery. Twenty-eight patients $(67 \%)$ underwent VPS treatment, with the remaining $14(33 \%)$ undergoing ETV with or without CPC (ETV \pm CPC). Within 12 months after initial hydrocephalus intervention, 14 patients (34\%) required revision (8 VPS and 6 ETV $\pm \mathrm{CPC}$ ). At the most recent follow-up, 21 patients $(50 \%)$ required a revision. The revision rate decreased as age increased. The overall infection rate was $5 \%$ (VPS $7 \%, 0 \% \mathrm{ETV} \pm \mathrm{CPC}$ ).

CONCLUSIONS This is the largest prospective study reported on children with craniosynostosis and hydrocephalus. Hydrocephalus in children with craniosynostosis most commonly occurs in syndromic patients and multisuture fusion. It is treated at varying ages; however, most patients undergo surgery for craniosynostosis prior to hydrocephalus treatment. While VPS treatment is performed more frequently, VPS and ETV are both reasonable options, with decreasing revision rates with increasing age, for the treatment of hydrocephalus associated with craniosynostosis. https://thejns.org/doi/abs/10.3171/2021.1.FOCUS20979

KEYWORDS craniosynostosis; hydrocephalus; endoscopic third ventriculostomy; ventriculoperitoneal shunt

ABBREVIATIONS CPC = choroid plexus cauterization; $\mathrm{ETV}=$ endoscopic third ventriculostomy; $\mathrm{ETV} \pm \mathrm{CPC}=\mathrm{ETV}$ with or without $\mathrm{CPC}$; FOHR = frontooccipital horn ratio; HCRN = Hydrocephalus Clinical Research Network; VPS = ventriculoperitoneal shunt.

SUBMITTED November 13, 2020. ACCEPTED January 6, 2021.

INCLUDE WHEN CITING DOI: 10.3171/2021.1.FOCUS20979. 
$\mathrm{P}$ ATIENTS with craniosynostosis, especially those with multisuture involvement, can develop hydrocephalus, including up to $100 \%$ of children with cloverleaf skull deformity. ${ }^{1-3}$ In these patients with multisuture craniosynostosis and progressive hydrocephalus, treatment is necessary to avoid neurological deterioration. Traditionally, ventriculoperitoneal shunt (VPS) insertion was the primary treatment for hydrocephalus in this population. Although the use of protocols and improved shunt equipment has led to fewer complications, VPS failures and infections remain a concern and require replacement of the VPS. ${ }^{4}$ In addition, VPS insertion is thought to potentially have a worsening effect on the patient's craniosynostosis. As noted by Di Rocco et al., "The insertion of an... CSF shunt in children with craniosynostosis may further aggravate the already impaired growth of the skull by counteracting the major factor for calvarial expansion - the tension exerted on the dura and skull by the intradural content. In a late phase, the chronic diversion of CSF and possibly an excessive decrease of ICP [intracranial pressure] due to the action of the CSF shunting device may result in craniocephalic disproportion due to both an early fusion of normal cranial sutures (secondary synostosis) and bone deposition at the inner surface of the calvarial bones." 5

Endoscopic third ventriculostomy (ETV), with or without choroid plexus cauterization $(\mathrm{CPC}$; ETV $\pm \mathrm{CPC})$, may be a viable alternative in this patient population, potentially leading to fewer revision surgeries. At present, there are very limited published data to address this highly important question. The aim of this study was to assess the viability and efficacy of ETV \pm CPC in the treatment of hydrocephalus in children with craniosynostosis, and to compare the success of ETV in this patient population with that of VPS insertion. However, patients presenting with both craniosynostosis and hydrocephalus tend to be less common, and any interpretation or conclusion would be limited by the small number of patients available at a single center. Therefore, the increased sample size available within the Hydrocephalus Clinical Research Network (HCRN) prospective Core Data Project (Registry) was queried. This study is the largest reporting of the effectiveness of utilizing VPS insertion or ETV to treat hydrocephalus in the pediatric craniosynostosis population.

\section{Methods}

This study utilizes the prospectively collected data within the HCRN Registry, augmented by retrospectively collected data specific to craniosynostosis type. The Registry is an ongoing registry containing clinical data pertaining to patients receiving neurosurgical services at each of the HCRN clinical sites. The study was approved by the institutional review board or research ethics board at each participating site as well as the Data Coordinating Center (DCC) at the University of Utah. All patients who underwent treatment for hydrocephalus associated with craniosynostosis were identified in the Registry. Patients were included if 1) they were in the Registry and had undergone VPS placement, ETV, or ETV + CPC; 2) they also had a diagnosis of craniosynostosis recorded in the Registry; 3 ) their records were available for review at the clinical site; and 4) they underwent a first permanent CSF diversion procedure occurring between March 2009 and December 2016. For the prospective cohort, data were collected by a dedicated HCRN research coordinator in the operating room, clinic, and ward settings. For the retrospective cohort, data were abstracted from electronic and paper patient medical charts and imaging records and entered into an electronic data capture system on a secure server at the DCC, where they were systematically queried for data quality. All patients were classified as having either single- or multiple-suture craniosynostosis, then by CSF diversion type. Descriptive statistics, demographics, and surgical outcomes were evaluated. Continuous variables are summarized as median (first quartile, third quartile) or mean (standard deviation). Categorical variables are reported as counts and percentages. The difference in survival of the first permanent CSF diversion between shunts and ETVs was analyzed using Kaplan-Meier curves and the log-rank test. Analyses were performed using SAS (version 9.4, SAS Institute).

The need and timing for surgical hydrocephalus treatment was based on a combination of physical examination findings (e.g., fontanelle characteristics, head circumference measurement, bone defects) and radiographic imaging (e.g., ventricular size, high frontooccipital horn ratio [FOHR]). The decision to perform VPS or ETV, with or without CPC, was made on a patient-by-patient case basis; discussion took place between the surgeon and the patient's family or caregiver regarding details of the procedure, as well as risks, benefits, and alternatives.

\section{Results}

In total, 42 patients underwent treatment for hydrocephalus associated with craniosynostosis. At the time of review, the HCRN Registry contained 6152 patients, with 46 patients $(0.75 \%)$ with the etiology of craniosynostosis. Of those 46 patients, 2 were removed from the paper cohort as their permanent procedure was not included in the Registry, and 2 others died before first craniosynostosis surgery. The overall patient demographics are illustrated in Table 1 . The median gestational age at birth was 39.0 weeks (IQR 38.0, 40.0), and the median birth weight was $3.2 \mathrm{~kg}$ (IQR 2.8,3.5). Females accounted for $55 \%(\mathrm{n}=23)$ of the cohort, and $60 \%(\mathrm{n}=25)$ were White. The majority $(\mathrm{n}=25,60 \%)$ had public primary insurance.

Treatment specifics are detailed in Table 2. Thirtythree patients (79\%) had multiple different sutures fused, and 9 had a single suture: 3 unicoronal (7\%), 3 sagittal (7\%), 2 lambdoidal (5\%), and 1 unknown (2\%). Syndromes were identified in 38 patients $(90 \%)$, with Crouzon syndrome being the most common $(n=16,42 \%)$. The median age at first craniosynostosis surgery was 0.6 years (IQR $0.3,1.7)$, with frontoorbital advancement $(\mathrm{n}=12,33 \%)$ and posterior cranial vault surgery $(\mathrm{n}=11,31 \%)$ being the most common craniofacial operations performed. The median age at hydrocephalus surgery was 1.2 years (IQR $0.5,2.5$ ) (median ages for VPS and ETV 1.3 and 1.1 years, respectively). Ten patients (28\%) received permanent hydrocephalus surgery before their first craniosynostosis 
TABLE 1. Patient demographics

\begin{tabular}{|c|c|c|c|}
\hline & \multicolumn{2}{|c|}{ Type of Initial CSF Diversion } & \multirow[b]{2}{*}{ Overall $(n=42)$} \\
\hline & Shunt $(n=28)$ & $\mathrm{ETV} \pm \mathrm{CPC}(\mathrm{n}=14)$ & \\
\hline Female sex & $15(53.6)$ & $8(57.1)$ & $23(54.8)$ \\
\hline \multicolumn{4}{|l|}{ Race } \\
\hline Asian & $3(10.7)$ & $0(0.0)$ & $3(7.1)$ \\
\hline Black or African American & $4(14.3)$ & $3(21.4)$ & $7(16.7)$ \\
\hline White & $16(57.1)$ & $9(64.3)$ & $25(59.5)$ \\
\hline Other & $1(3.6)$ & $1(7.1)$ & $2(4.8)$ \\
\hline Unknown or not reported & $4(14.3)$ & $1(7.1)$ & $5(11.9)$ \\
\hline \multicolumn{4}{|l|}{ Ethnicity } \\
\hline Not Hispanic or Latino & $21(75.0)$ & $11(78.6)$ & $32(76.2)$ \\
\hline Hispanic or Latino & $4(14.3)$ & $2(14.3)$ & $6(14.3)$ \\
\hline Unknown or not reported & $3(10.7)$ & $1(7.1)$ & $4(9.5)$ \\
\hline \multicolumn{4}{|l|}{ Primary insurance classification } \\
\hline Public & $17(60.7)$ & $8(57.1)$ & $25(59.5)$ \\
\hline Private & $11(39.3)$ & $5(35.7)$ & $16(38.1)$ \\
\hline Self-pay & $0(0.0)$ & $1(7.1)$ & $1(2.4)$ \\
\hline Median gestational age at birth, wks* & $39.0[37.0,40.0]$ & $38.5[38.0,40.0]$ & $39.0[38.0,40.0]$ \\
\hline Median birth weight, kg† & $3.2[2.8,3.5]$ & $3.3[2.8,3.4]$ & $3.2[2.8,3.5]$ \\
\hline
\end{tabular}

Values are presented as the number of patients (\%) or the median [IQR].

* Missing for 13 patients.

† Missing for 16 patients.

surgery. Twenty-eight patients (67\%) underwent VPS insertion, with the remaining 14 (33\%) undergoing ETV. Of note, 4 patients in the ETV group underwent CPC as part of the procedure. The mean follow-up from CSF diversion treatment was 5.2 years (range $0.1-9.7$ years).

Within 12 months after initial hydrocephalus intervention, 14 patients (34\%) required revision (8 with initial VPS insertion and 6 with initial ETV \pm CPC) (Table 3). More than two-thirds of the patients were younger than 2 years of age when they received hydrocephalus treatment $(<1$ year, $\mathrm{n}=16,38 \% ; 1-2$ years, $\mathrm{n}=14,33 \%)$. The rate of revisions decreased as age increased $(<1$ year, $67 \%, 10 / 15$ patients; $1-2$ years, 21\%, 3/14 patients; $3-4$ years, 20\%, 1/5 patients; 5-10 years, 0\%, 0/7 patients). At the most recent follow-up, revision had been required for 52\% (14/25 VPS, $6 / 13$ ETV \pm CPC) with a syndromic diagnosis, compared with $25 \%$ (1/3 VPS, 0/1 ETV \pm CPC) for patients without a syndrome. Multisuture craniosynostosis was associated with a failure rate of 58\% (14/22 VPS, 5/11 ETV \pm CPC), while single-suture craniosynostosis was associated with a failure rate of $22 \%$ (1/6 VPS, $1 / 3 \mathrm{ETV} \pm$ CPC). Overall, a total of 21 patients (50\%) required revision. Figure 1 shows the Kaplan-Meier curves for time to failure of the initial CSF diversion procedure between VPS and ETV \pm CPC. Overall, there were no significant differences in survival probability of the VPS or ETV procedure compared with each other $(\mathrm{p}=0.4298)$.

Complications are shown in Table 4. CSF leaks were observed in $1(4 \%), 1(10 \%)$, and $0(0 \%)$ of patients in the VPS, ETV, and ETV + CPC groups, respectively. There was 1 ETV patient (7\%) with pseudomeningocele who required a reoperation, and 1 ETV patient (10\%) had postop- erative seizures. Overall, the infection rate for VPS insertion was $7 \%(\mathrm{n}=2 / 28)$, and for ETV $\pm \mathrm{CPC}$ it was $0 \%(\mathrm{n}=$ $0 / 14)$. None of the patients had a new neurological deficit or stroke after surgical intervention $(n=0 / 42)$.

\section{Discussion}

Hydrocephalus is commonly seen in patients with complex, syndromic, and multisuture craniosynostosis.,2,6-9 Traditionally, VPS insertion was the treatment of choice for hydrocephalus in this population, despite the potential for worsening the craniofacial deformity due to both an early fusion of normal cranial sutures (secondary synostosis) and bone deposition at the inner surface of the calvarial bones. ${ }^{5}$ It could also inhibit growth of the skull after the craniofacial reconstruction. Recently, ETV has been more frequently used in children with varying etiologies of hydrocephalus..$^{10,11}$ This study suggests that VPS and ETV have similar revision rates and are both reasonable options for the treatment of hydrocephalus associated with craniosynostosis. Overall, $50 \%$ of patients required revision of their primary hydrocephalus treatment, although there were fewer failures seen in the nonsyndromic and single-suture groups compared to the syndromic and multisuture groups. This could be a result of the more complex patients having more challenging anatomy for the surgical procedures; having pathophysiological differences in regard to the true underlying etiology of the hydrocephalus; or requiring more surgical procedures in general, which expose the VPS to manipulation, failure, or infection. The small numbers in these subgroups, however, limit detailed analysis and comparison. 
TABLE 2. Treatment details

\begin{tabular}{|c|c|c|c|}
\hline & \multicolumn{2}{|c|}{ Type of Initial CSF Diversion } & \multirow[b]{2}{*}{ Overall $(n=42)$} \\
\hline & Shunt $(n=28)$ & $\mathrm{ETV} \pm \mathrm{CPC}(\mathrm{n}=14)$ & \\
\hline \multicolumn{4}{|l|}{ Sutures fused } \\
\hline Sagittal & $1(3.6)$ & $2(14.3)$ & $3(7.1)$ \\
\hline Coronal & $3(10.7)$ & $0(0.0)$ & $3(7.1)$ \\
\hline Lambdoidal & $2(7.1)$ & $0(0.0)$ & $2(4.8)$ \\
\hline Multiple different sutures* & $22(78.6)$ & $11(78.6)$ & $33(78.6)$ \\
\hline Unknown & $0(0.0)$ & $1(7.1)$ & $1(2.4)$ \\
\hline \multicolumn{4}{|l|}{ Craniosynostosis syndrome $†$} \\
\hline Crouzon & $13(52.0)$ & $3(23.1)$ & $16(42.1)$ \\
\hline Apert & $3(12.0)$ & $1(7.7)$ & $4(10.5)$ \\
\hline Pfeiffer & $5(20.0)$ & $3(23.1)$ & $8(21.1)$ \\
\hline Other $\ddagger$ & $4(16.0)$ & $6(46.2)$ & $10(26.3)$ \\
\hline Median corrected age at 1st craniosynostosis surgery, yrs§ & $0.5[0.2,4.1]$ & $0.6[0.2,0.7]$ & $0.5[0.2,1.7]$ \\
\hline Median age at 1st craniosynostosis surgery, yrs & $0.8[0.3,1.7]$ & $0.6[0.3,1.8]$ & $0.6[0.3,1.7]$ \\
\hline \multicolumn{4}{|l|}{ Type of 1st craniosynostosis surgery } \\
\hline Strip craniectomy & $3(11.5)$ & $1(10.0)$ & $4(11.1)$ \\
\hline Frontoorbital advancement & $10(38.5)$ & $2(20.0)$ & $12(33.3)$ \\
\hline Total cranial vault & $5(19.2)$ & $3(30.0)$ & $8(22.2)$ \\
\hline Posterior cranial vault & $7(26.9)$ & $4(40.0)$ & $11(30.6)$ \\
\hline Other & $1(3.8)$ & $0(0.0)$ & $1(2.8)$ \\
\hline Additional craniosynostosis surgeries required & $13(50.0)$ & $4(40.0)$ & $17(47.2)$ \\
\hline Median age at initial CSF diversion, yrs & $1.3[0.5,2.1]$ & $1.1[0.4,3.3]$ & $1.2[0.5,2.5]$ \\
\hline Median duration of surgery for 1st permanent CSF diversion, mins & $48.5[39.0,56.0]$ & $93.0[61.0,134.0]$ & $52.0[43.0,68.0]$ \\
\hline CPC performed & & $4(40.0)$ & $4(40.0)$ \\
\hline 1st permanent CSF diversion prior to 1st craniosynostosis surgery & $9(34.6)$ & $1(10.0)$ & $10(27.8)$ \\
\hline Use of postop helmet & $2(7.7)$ & $3(30.0)$ & $5(13.9)$ \\
\hline Median helmet duration, mos & $3.5[3.0,4.0]$ & $12.0[5.0,18.0]$ & $5.0[4.0,12.0]$ \\
\hline Median time from 1st permanent CSF diversion to discharge, days & $2.0[1.0,3.0]$ & $3.0[1.0,7.0]$ & $2.0[1.0,5.0]$ \\
\hline \multicolumn{4}{|c|}{$\begin{array}{l}\text { Values are presented as the number of patients (\%) or the median [IQR]. } \\
\text { *Eleven were bilateral. } \\
\text { † Four patients did not have a syndrome. } \\
\text { † Other syndromes include bilateral lambdoid and sagittal, Beare-Stevenson, cloverleaf, microcephaly osteopathic primordial dwarfism, familial FGFR-associated } \\
\text { craniosynostosis, Garland, Hurler, and Loeys-Dietz in } 1 \text { patient each; and unknown in } 2 \text { patients. } \\
\text { § Six patients did not undergo craniosynostosis surgery and are not included in summaries related to craniosynostosis surgeries. Of those who underwent craniosynos- } \\
\text { tosis surgery, } 8 \text { patients are missing the corrected age at the first craniosynostosis surgery. }\end{array}$} \\
\hline
\end{tabular}

In general, this study reports similar VPS and ETV outcomes compared with patients with other hydrocephalus etiologies. In a large, prospective multicenter study, the HRCN reported a $33.3 \%$ VPS failure rate with a shorter overall follow-up. ${ }^{12}$ The VPS infection rate was also simi$\operatorname{lar}(7.6 \%$ compared with $7 \%$ in the current study). ETV outcomes are also similarly reported in the literature with an ETV + CPC success rate of $46 \%$ (younger pediatric patients, median age 6.3 weeks) at 1 year and an ETV success rate of $61.7 \%$ (older pediatric population, mean age 6.9 years) at 1 year. $^{10,11}$ These large studies also report similar complications of the hydrocephalus procedures. Furthermore, as seen in the current study, success rates of both VPS and ETV \pm CPC improve with increasing age.

The etiology for hydrocephalus for children with craniosynostosis is not well understood. These patients often have craniocerebral disproportion with a small posterior fossa, which could lead to CSF outflow obstruction. However, more recent literature has investigated the venous anomalies seen in these patients as another cause of hydrocephalus and intracranial hypertension. Although the direct mechanism between these abnormalities and hydrocephalus remains unresolved,${ }^{13}$ there has been a reported association between venous anomalies with elevated intracranial pressure and shunted hydrocephalus. ${ }^{14}$ Most likely, the development of hydrocephalus in the complex craniosynostosis population is multifactorial.

ETV has been utilized in other populations with craniocerebral disproportion and jugular venous stenosis, such as those with achondroplasia. In two case reports, ${ }^{15,16}$ 5 children with hydrocephalus and achondroplasia were successfully treated with ETV. Although patients with achondroplasia and craniosynostosis are not the same population, there may be overlap in the mechanism of hy- 


\begin{tabular}{|c|c|c|c|}
\hline & \multicolumn{3}{|c|}{ No. of Patients (\%) } \\
\hline & \multicolumn{2}{|c|}{ Type of Initial CSF Diversion } & \multirow[b]{2}{*}{ Overall $(n=42)$} \\
\hline & Shunt $(n=28)$ & $\mathrm{ETV} \pm \mathrm{CPC}(\mathrm{n}=14)$ & \\
\hline \multicolumn{4}{|l|}{ Age at initial CSF diversion (yrs) } \\
\hline$<1$ & $11(39.3)$ & $5(53.7)$ & $16(38.1)$ \\
\hline $1-2$ & $11(39.3)$ & $3(21.4)$ & $14(33.3)$ \\
\hline $3-4$ & $2(7.1)$ & $3(21.4)$ & $5(11.9)$ \\
\hline \multirow[t]{3}{*}{$5-10$} & $4(14.3)$ & $3(21.4)$ & $7(16.7)$ \\
\hline & \multicolumn{2}{|c|}{ Type of Initial CSF Diversion } & \\
\hline & Shunt $(n=15)$ & $\mathrm{ETV} \pm \mathrm{CPC}(\mathrm{n}=6)$ & Overall $(n=21)$ \\
\hline \multirow[t]{3}{*}{ Revision occurred w/in yr of initial CSF diversion* } & $8(53.3)$ & $6(100.0)$ & $14(66.7)$ \\
\hline & \multicolumn{2}{|c|}{ Revision Occurred w/in Year of Initial CSF Diversion } & \\
\hline & No $(n=7)$ & Yes $(n=14)$ & Overall $(n=21)$ \\
\hline \multicolumn{4}{|l|}{ Age at initial CSF diversion (yrs) ${ }^{*} \dagger$} \\
\hline$<1$ & $0(0.0)$ & $10(100.0)$ & 10 \\
\hline $1-2$ & $5(62.5)$ & $3(37.5)$ & 8 \\
\hline $3-4$ & $1(50.0)$ & $1(50.0)$ & 2 \\
\hline $5-10$ & $1(100.0)$ & $0(0.0)$ & 1 \\
\hline
\end{tabular}

* Only includes patients who ever had a revision and with at least 1 year of follow-up.

$\dagger$ Percentages are calculated as row percentages.

drocephalus development, and both disorders may be successfully treated with ETV.

As there appears to be no major difference in success rates between VPS and ETV, ETV could be used more frequently in children with hydrocephalus and craniosynostosis. It is also our experience that some families would prefer that an ETV \pm CPC be attempted as an initial hydrocephalus operation for the possibility of achieving shunt freedom. Minimizing the use of VPS insertions also has the advantage of not leaving hardware within the patients, which could be especially important in patients who undergo further cranial vault reconstruction in the future. Often, the VPS needs to be moved to complete the craniofacial surgery, and exposure of the VPS poses a risk for shunt infection. An ETV performed prior to the craniofacial operation would not have this risk.

For both multisuture and single-suture patients, the decision to treat and the timing of hydrocephalus treatment must be carefully planned. Each treatment plan should be made on a case-by-case basis, taking into consideration factors such as the overall condition of the child, the severity of the craniosynostosis and hydrocephalus, and the planned craniosynostosis surgery. Knowledge of the underlying syndrome is also important, as hydrocephalus is more commonly seen in certain syndromes (Crouzon, Pfeiffer) than in others (Apert). For example, nonprogressive ventriculomegaly is commonly found in patients with Apert syndrome. Also, identification of characteristics that might potentially increase intracranial pressure must be noticed before hydrocephalus treatment is undertaken, especially in these patients. For this reason, the need and timing for surgical hydrocephalus treatment were based on a combination of physical examination findings (e.g., fontanelle characteristics, head circumference measurement, bone defects, copper-beaten appearance of the skull) and radiographic imaging findings (e.g., progressive ventricular size, FOHR). In a minority of the patients with severe hydrocephalus, the hydrocephalus treatment was performed prior to the craniofacial operation. If left untreated, the increased intracranial pressure could lead not only to neurological deficit and morbidity but also to significant thinning of the bone, rendering the craniofacial reconstruction more difficult. However, most of the patients required hydrocephalus treatment after the cra-

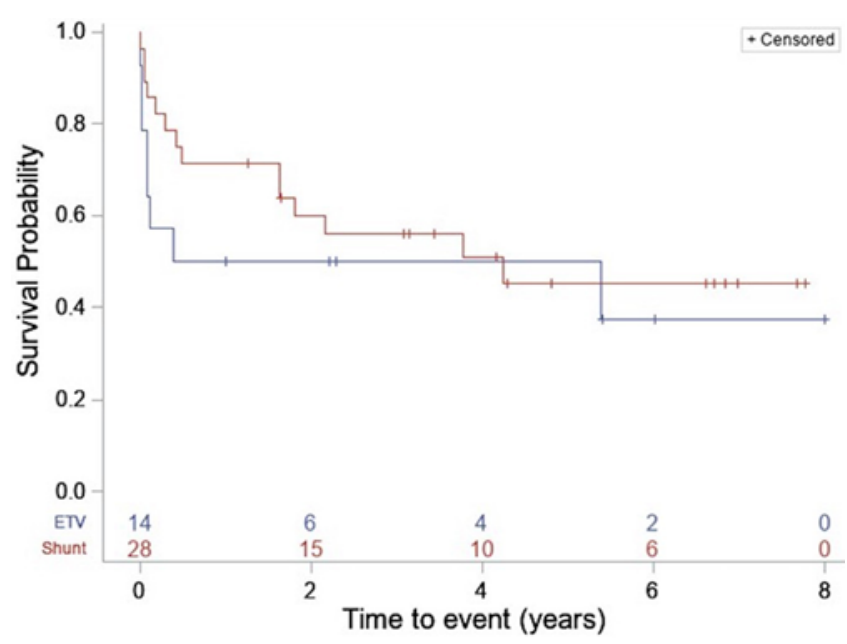

FIG. 1. Kaplan-Meier curve analysis showing time to failure of the initial CSF diversion procedure between VPS insertion and ETV \pm CPC. 


\begin{tabular}{|c|c|c|c|}
\hline & \multicolumn{3}{|c|}{ No. of Patients (\%) } \\
\hline & Shunt $(n=28)$ & ETV \pm CPC $(n=14)$ & Overall $(n=42)$ \\
\hline CSF leak & $1(3.6)$ & $1(7.1)$ & $2(4.8)$ \\
\hline Wound, pseudomeningocele & $0(0.0)$ & $1(7.1)$ & $1(2.4)$ \\
\hline Wound, superficial infection/dehiscence & $2(7.1)$ & $0(0.0)$ & $2(4.8)$ \\
\hline CSF infection & $1(3.6)$ & $0(0.0)$ & $1(2.4)$ \\
\hline Postop seizures & $0(0.0)$ & $1(7.1)$ & $1(2.4)$ \\
\hline New neurological deficit/strokes & $0(0.0)$ & $0(0.0)$ & $0(0.0)$ \\
\hline
\end{tabular}

Summaries reflect complications that occurred from the time of the first permanent procedure to the next procedure, or 6 months postoperatively, whichever came first.

niosynostosis surgery, avoiding the issues discussed earlier. The decision to perform VPS or ETV \pm CPC was also made on a case-by-case basis considering surgeon expertise, patient anatomy, and discussion between the surgeon and the patient's family or caregiver.

This study is the largest cohort of patients with hydrocephalus due to craniosynostosis treated by VPS or ETV, utilizing a large, multicenter, prospectively collected registry. However, it is not without limitations. Craniosynostosis data are reviewed retrospectively, and individual case details, including specific details determining the need for hydrocephalus treatment, are limited by the input to the Registry. Despite being the largest cohort of these patients, small numbers in the nonsyndromic and single-suture subgroups limit detailed analysis and conclusions. Furthermore, data on craniofacial complications or need for repeat craniofacial surgery are not included in the analysis. A larger cohort of patients with increased follow-up is needed to validate and advance the findings of this study.

\section{Conclusions}

This is the largest prospective study reported on children with craniosynostosis and hydrocephalus. Hydrocephalus in children with craniosynostosis frequently occurs in syndromic patients and those with multisuture fusion. It is treated at varying ages; however, most children have surgery for craniosynostosis prior to hydrocephalus treatment. While VPS insertion is performed more frequently, VPS and ETV are both reasonable options, with decreasing revision rates with increasing age, for the treatment of hydrocephalus associated with craniosynostosis.

\section{Appendix}

\section{Hydrocephalus Clinical Research Network Members}

The HCRN currently consists of the following clinical centers and investigators: Primary Children's Hospital, University of Utah (J. Kestle); Children's Hospital of Alabama, University of Alabama at Birmingham (C. Rozzelle); Hospital for Sick Children, University of Toronto (J. Drake, A. Kulkarni); Texas Children's Hospital, Baylor College of Medicine (W. Whitehead); Seattle Children's Hospital, University of Washington (S. Browd, T. Simon, J. Hauptman); Children's Hospital of Pittsburgh, University of Pittsburgh (I. Pollack); St. Louis Children's Hospital, Washington University in St. Louis (D. Limbrick); Monroe Carell Jr. Children's Hospital at Vanderbilt, Vanderbilt University Medical Center (J. Wellons, R. Naftel, C. Shannon); British Columbia Children's Hospital, University of British Columbia (M. Tamber,
P. McDonald); Alberta Children's Hospital, University of Calgary (J. Riva-Cambrin); The Johns Hopkins Hospital (E. Jackson); Children's Hospital of Los Angeles (M. Krieger); Children's Hospital Colorado (T. Hankinson); Nationwide Children's Hospital (J. Pindrik); HCRN Data Coordinating Center, Department of Pediatrics, University of Utah (R. Holubkov).

\section{Acknowledgments}

The HCRN has been funded by the National Institute of Neurological Disorders and Stroke (NINDS grant no. 1RC1NS06894301), Patient Centered Outcome Research Institute (PCORI contract no. CER-1403-13857), The Gerber Foundation (reference no. 16923638), private philanthropy, and the Hydrocephalus Association.

We would like to thank our colleagues for their past and ongoing support of HCRN: D. Brockmeyer, M. Walker, R. Bollo, S. Cheshier, J. Blount, J. Johnston, B. Rocque, L. Ackacpo-Satchivi, J. Oakes, P. Dirks, J. Rutka, M. Taylor, D. Curry, R. Dauser, A. Jea, S. Lam, H. Weiner, T. Luerssen, R. Ellenbogen, J. Ojemann, A. Lee, A. Avellino, S. Greene, E. Tyler-Kabara, T. Abel, T. S. Park, M. Smyth, N. Tulipan, A. Singhal, P. Steinbok, D. Cochrane, W. Hader, C. Gallagher, M. Benour, E. Kiehna, J. G. McComb, A. Robison, A. Alexander, M. Handler, B. O'Neill, C. Wilkinson, L. Governale, J. Leonard, E. Sribnick, E. Ahn, A. Cohen, M. Groves, and S. Robinson.

In addition, our work would not be possible without the outstanding support of the dedicated personnel at each clinical site and the Data Coordinating Center. Special thanks goes to J. Clawson, P. Martello, N. Tattersall, and T. Bach (Salt Lake City); A. Arynchyna and A. Bey (Birmingham); H. Ashrafpour, M. Lamberti-Pasculli, and L. O'Connor (Toronto); E. Sanchez, S. Martinez, and S. Ryan (Houston); A. Anderson and G. Bowen (Seattle); K. Diamond and A. Luther (Pittsburgh); H. Botteron, D. Morales, M. Gabir, D. Berger, and D. Mercer (St. Louis); J. Stoll, D. Dawson, and S. Gannon (Nashville); A. Cheong and R. Hengel (British Columbia); R. Rashid and S. Ahmed (Calgary); R. Daniel and A. Loudermilk (Baltimore); N. Rea and C. Cook (Los Angeles); S. Staulcup (Colorado); A. Sheline (Columbus); and M. Langley, V. Wall, N. Nunn, H. Jensen, V. Freimann, L. Herrera, and B. Miller (Utah Data Coordinating Center).

\section{References}

1. Cinalli G, Sainte-Rose C, Kollar EM, et al. Hydrocephalus and craniosynostosis. J Neurosurg. 1998;88(2):209-214.

2. Collmann H, Sörensen N, Krauss J. Hydrocephalus in craniosynostosis: a review. Childs Nerv Syst. 2005;21(10): 902-912.

3. Golabi M, Edwards MS, Ousterhout DK. Craniosynostosis and hydrocephalus. Neurosurgery. 1987;21(1):63-67.

4. Khan F, Shamim MS, Rehman A, Bari ME. Analysis of factors affecting ventriculoperitoneal shunt survival in pediatric patients. Childs Nerv Syst. 2013;29(5):791-802. 
5. Di Rocco F, Jucá CE, Arnaud E, et al. The role of endoscopic third ventriculostomy in the treatment of hydrocephalus associated with faciocraniosynostosis. J Neurosurg Pediatr. 2010; 6(1):17-22.

6. Blaser SI, Padfield N, Chitayat D, Forrest CR. Skull base development and craniosynostosis. Pediatr Radiol. 2015; 45(suppl 3):S485-S496.

7. Hayward R, Gonsalez S. How low can you go? Intracranial pressure, cerebral perfusion pressure, and respiratory obstruction in children with complex craniosynostosis. J Neurosurg. 2005;102(1)(suppl):16-22.

8. Marucci DD, Dunaway DJ, Jones BM, Hayward RD. Raised intracranial pressure in Apert syndrome. Plast Reconstr Surg. 2008;122(4):1162-1168.

9. Renier D, Sainte-Rose C, Marchac D, Hirsch JF. Intracranial pressure in craniostenosis. J Neurosurg. 1982;57(3):370-377.

10. Kulkarni AV, Riva-Cambrin J, Holubkov R, et al. Endoscopic third ventriculostomy in children: prospective, multicenter results from the Hydrocephalus Clinical Research Network. $J$ Neurosurg Pediatr. 2016;18(4):423-429.

11. Riva-Cambrin J, Kestle JRW, Rozzelle CJ, et al. Predictors of success for combined endoscopic third ventriculostomy and choroid plexus cauterization in a North American setting: a Hydrocephalus Clinical Research Network study. J Neurosurg Pediatr. 2019;24(2):128-138.

12. Riva-Cambrin J, Kestle JR, Holubkov R, et al. Risk factors for shunt malfunction in pediatric hydrocephalus: a multicenter prospective cohort study. J Neurosurg Pediatr. 2016; 17(4):382-390.

13. Ghali GZ, Zaki Ghali MG, Ghali EZ, et al. Intracranial venous hypertension in craniosynostosis: mechanistic underpinnings and therapeutic implications. World Neurosurg. 2019;127:549-558.

14. Copeland AE, Hoffman CE, Tsitouras V, et al. Clinical significance of venous anomalies in syndromic craniosynostosis. Plast Reconstr Surg Glob Open. 2018;6(1):e1613.
15. Etus V, Ceylan S. The role of endoscopic third ventriculostomy in the treatment of triventricular hydrocephalus seen in children with achondroplasia. J Neurosurg. 2005;103(3) (suppl):260-265.

16. Swift D, Nagy L, Robertson B. Endoscopic third ventriculostomy in hydrocephalus associated with achondroplasia. $J$ Neurosurg Pediatr. 2012;9(1):73-81.

\section{Disclosures}

Dr. Hauptman: consultant for Medtronic. Dr. Limbrick: support of non-study-related clinical or research effort from Medtronic and Microbot Medical.

\section{Author Contributions}

Conception and design: Bonfield, Kestle, Wellons. Acquisition of data: all authors. Analysis and interpretation of data: Bonfield, Shannon, Reeder. Drafting the article: Bonfield, Shannon, Reeder, Naftel, Kestle, Wellons. Critically revising the article: all authors. Reviewed submitted version of manuscript: Bonfield. Approved the final version of the manuscript on behalf of all authors: Bonfield. Study supervision: Kestle, Wellons.

\section{Correspondence}

Christopher M. Bonfield: Vanderbilt University Medical Center, Nashville, TN. chris.bonfield@vumc.org. 